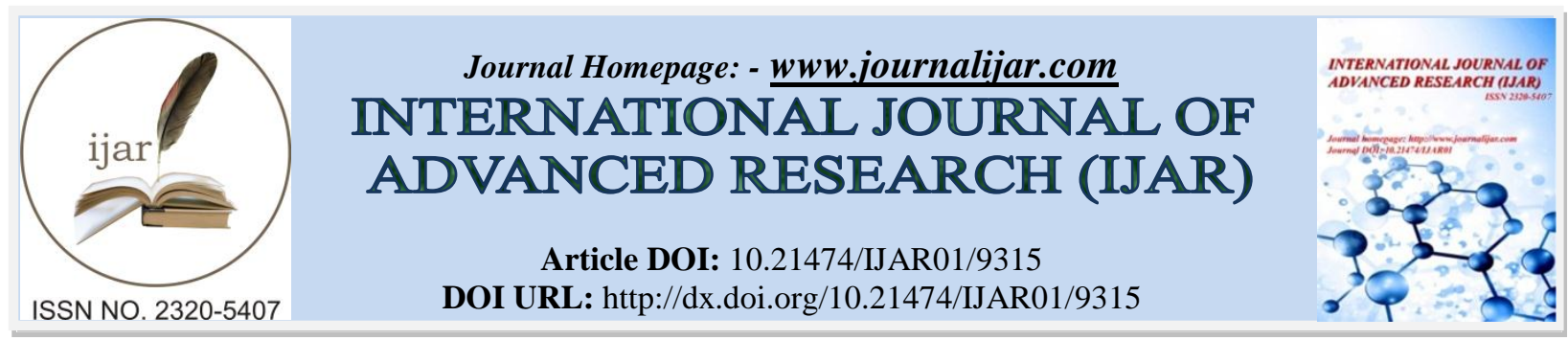

RESEARCH ARTICLE

\title{
SYMMETRICAL ANALYSIS: AN X-RAY STUDY OF TALUS WITH ITS PHYSIOLOGICAL CORRELATION.
}

Kavya $^{1}$, Sowmya Panju ${ }^{2}$ and Ashwin Kumar ${ }^{3}$.

1. Assistant professor, Department of Anatomy, Bowring and Lady Curzon medical college and research institute, Bangalore.

2. Assistant professor, Department of Physiology, Bowring and Lady Curzon medical college and research institute, Bangalore.

3. Professor \& HOD, Department of Radiology, Kasturba Medical college, Mangalore.

\section{Manuscript Info}

Manuscript History

Received: 15 April 2019

Final Accepted: 17 May 2019

Published: June 2019

Key words:-

Talus, foot, X-ray.

\section{Abstract}

Out of all the bones in the foot involved in movement, talus has constantly formed the sole link between the leg and the rest of the foot. Differences have been noted between or within body structures within an individual (intra-individual variation) with regard to the size and shape. Talus helps in receiving the body weight and transmitting it to the plantar below. The dome of the talus articulates with the tibia and fibula, and has a key role in ankle motion and also supports the axial load during weight bearing.

Aim: To study the talar symmetry and to identify the incidence, possible etiological factors and its clinical significance.

Materials and methods:This study included 96 healthy normal subjects whose age and sex are known who were scheduled for anteroposterior and lateral plain ankle radiographs because of clinical hind foot symptoms. Measurements such as length, breadth, height and volume were noted down for a period of 2 years to the Department of Radiology, K.M.C. Mangalore.

Result: In this study the measurement of right tali were higher than left except for breath of the body of the tali.

Conclusion: The human foot is called upon to support the weight of the body when standing and to provide a smooth functioning interface between the body and the ground during gait. Its function depends on relatively complex mechanical interactions of numerous small bones; pain free motion of small joints, an intact soft tissue envelope, including the plantar pad; and an appropriate secondary feedback ${ }^{32}$.

Copy Right, IJAR, 2019,. All rights reserved.

\section{Introduction:-}

Talus is a main tarsal bone that connects bones of the leg with the bones of the foot. It has neither muscular attachments nor the tendinous attachments ${ }^{1}$. The talus (astragalus) sits at the top of the tarsal skeleton ${ }^{3}$. Head is ovoid and convex; its long axis is also inclined inferiomedially to articulate with the proximal navicular surface. No muscle is attached to the talus, however many ligaments confer the stability to the joints ${ }^{2}$. Blood supply to the talus

Corresponding Author:-Sowmya Panju

Address:- Assistant professor, Department of Physiology, Bowring and Lady Curzon medical college and research institute, Bangalore. 
is rather tenuous because of the lack of muscle attachments. The extra osseous blood supply is via the posterior tibial, dorsalis pedis and fibular arteries ${ }^{2}$.

Talus helps in receiving the body weight and transmitting it to the plantar below. The dome of the talus articulates with the tibia and fibula, and has a key role in ankle motion and also supports the axial load during weight bearing. The subtalar joint is a simple single axis joint which behaves like a oblique hinge. The average inclination of the subtalar joint axis is 42 degree from the horizontal in a sagittal plane and 16 degree from the longitudinal axis of the foot in the transverse plane thus; the axis is aligned in an oblique direction. The oblique orientation of the axis allows it to traverse all three cardinal planes and movement about the axis is termed "triplane motion." It is important to note that these motions occur about the subtalar joint axis only and do not include talocural or midtarsal joint motions. When the foot is weight bearing in stance phase, pronation and supination of the subtalar joint continue to occur. In an open kinetic chain, minimal movement occurs between the talus and the calcaneus as motion occurs distal to the subtalar joint axis. In a closed kinetic chain, movement occurs both proximal and distal to the axis of the subtalar joint, i.e., leg rotation and foot movement, respectively. The direct effect of subtalar joint is to create an immediate shortening of the lower extremity at heel strike, thus providing a small degree of shock absorption $^{3}$.

Aim:

To study the talar symmetry and to identify the incidence, possible etiological factors and its clinical significance.

\section{Materials and methods:-}

This study included 96 healthy normal subjects whose age and sex are known who were referred as patients to the radiology department, for an ankle x-ray (anterior posterior and laterl) with hind foot disorder. Ankle joint radiograph having talar and subtalar pathology were excluded and normal ankle radiograph were included from the study

The data was collected for a period of 2 years in the Department of Radiology, K.M.C. Mangalore.

Study sample size calculation: With $95 \%$ Confidence level and $80 \%$ Power, a sample size of 96 was calculated by using the formula,

Sample size $(\mathbf{n})=\mathbf{2} \times\left(\mathbf{Z \alpha}+\underset{\delta^{2}}{\delta^{2}} \times \sigma^{2}\right.$

The data collected was tabulated in Microsoft excel worksheet and computer- based analysis was done using the statistical package for social sciences software IBM SPSS 20.0 (Chicago, IL, USA) for windows.

The general descriptive statistics was done for all the parameters providing means and standard deviations (S.D) separately.

Student's t test was used to check whether significant differences exist $(\mathrm{p} \leq 0.05)$ between right and left mean measurements.

\section{Measurements of dimensions on radiograph:}

On anterior posterior radiograph

1. Breadth of the body of talus- the distance between the medial and the lateral surface. On lateral radiograph

2. Length of talus- the distance between the foremost part of the rounded articular surface of the head to the apex of the lateral tubercle.

3. Height of talus- the distance between the superior and inferior surface.

4. Volume is calculated by multiplying the value of length, breath and height.

\section{Results \& observation:-}

Table 1:-Difference between Parameters of Talus based on side (Radiograph)

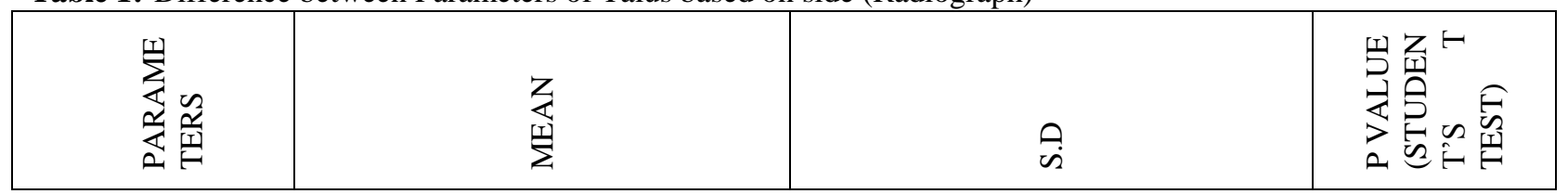




\begin{tabular}{|c|c|c|c|c|c|}
\hline & 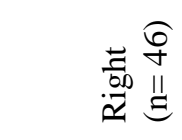 & 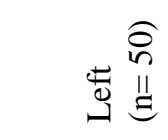 & 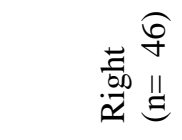 & 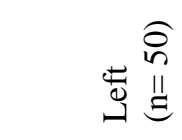 & \\
\hline 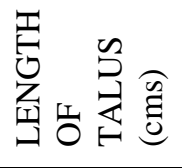 & 5.7359 & 5.6306 & 0.6075 & 0.5331 & 0.368 \\
\hline 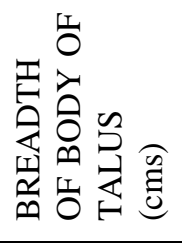 & 3.0833 & 3.0990 & 0.4081 & 0.4238 & 0.854 \\
\hline 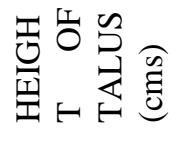 & 3.2280 & 3.2248 & 0.6179 & 0.6078 & 0.979 \\
\hline 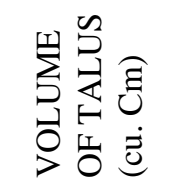 & 57.8570 & 57.2086 & 16.9943 & 17.8569 & 0.856 \\
\hline
\end{tabular}

Average length of right talus is $5.7359 \pm 0.6075 \mathrm{cms}$. The average length of left is $5.6306 \pm 0.5331 \mathrm{cms}$. Average breadth of the body of right talus is $3.0833 \pm 0.4081 \mathrm{cms}$. The average breadth of body of left talus is $3.0990 \pm$ $0.4238 \mathrm{cms}$. Average height of right talus is $3.2280 \pm 0.6179 \mathrm{cms}$. The average height of left talus is $3.2248 \pm$ $0.6078 \mathrm{cms}$. Average volume of right talus is $57.8570 \pm 16.9947 \mathrm{cu} \mathrm{cm}$. The average volume of left talus is 57.2086 $\pm 17.8569 \mathrm{cu} \mathrm{cm}$. The $\mathrm{P}$ values for length, breath, height and volume were $0.368,0.850,0.979$ and 0.856 respectively, which are statistically insignificant this showed that both the talus were symmetrical.

\section{Discussion:-}

The human foot is called upon to support the weight of the body when standing and to provide a smooth functioning interface between the body and the ground during gait. Its function depends on relatively complex mechanical interactions of numerous small bones; pain free motion of small joints, an intact soft tissue envelop, including the plantar pad; and an appropriate secondary feedback ${ }^{4}$. It is important to analyze the anatomical features of talus that predispose it to complex injury. Restoration of the normal anatomy is very important to prevent development of secondary pathology and morbidity.

In the present study average length of the right talus on radiograph are $\mathbf{5 . 7 3 5 9} \mathbf{~ c m s}$ and the left talus is $\mathbf{5 . 6 3 0 6} \mathbf{~ c m s}$ respectively. It has been observed that right talus were longer than the left. According to Ari I, Kafa IM ${ }^{5}$, the mean values were 5.72 for right side and 5.64 for left side, which were slight higher as compared to the present study. In a study conducted by Mahato NK, Murthy SN ${ }^{6}$ the length varied from $5.57 \mathrm{cms}$ on right side and $5.58 \mathrm{cms}$ on left side, another study by Otag I, Cimen $\mathrm{M}^{7}$ the length of right tali measured $5.594 \mathrm{cms}$ and left tali measured 5.654 $\mathrm{cms}$ and a similar study by Gautham $\mathrm{K} \mathrm{et} \mathrm{al}^{8}$ the length varied from 5.23 on right and 5.29 in left side.

The average breath of right talus on radiographs is $\mathbf{3 . 0 8 3 3} \mathbf{~ c m s}$ and the left talus is $\mathbf{3 . 0 9 9 0}$ cms respectively. It has been observed that left talus was broader than the right. In a similar study conducted by Ari I, Kafa IM ${ }^{5}$, the mean values were 4.91 for right side and 4.69 for left side, which were higher to the present study. According to a study conducted by Mahato NK, Murthy $\mathrm{SN}^{6}$ the breath varied from $2.99 \mathrm{cms}$ on right side and $3.03 \mathrm{cms}$ on left side and a study conducted by Gautham $\mathrm{K}$ et al ${ }^{8}$ the breath varied from $3.79 \mathrm{cms}$ on right and 3.68 on the left side. In a radiological study by Kuo $\mathrm{CC}$ et al ${ }^{9}$ the talar breath varied from $2.63 \mathrm{cms}$ to $2.96 \mathrm{cms}$ with an average of $2.99 \mathrm{cms}$, which is slightly lower than the values of the present study. 
The average height of right talus was $3.2280 \mathrm{cms}$ and left talus is $\mathbf{3 . 2 2 4 8} \mathbf{~ c m s}$ respectively. It has been observed that right talus was taller than the left by $0.0032 \mathrm{cms}$. In another study by Otag I, Cimen $\mathrm{M}^{7}$ observed that the height of right tali measured $3.149 \mathrm{cms}$ and left tali measured $3.215 \mathrm{cms}$.

In the present study the average volume of right talus on radiographs is $\mathbf{5 7 . 8 5 7 0} \mathbf{c u} \mathbf{~ c m s}$ and left talus is $\mathbf{5 7 . 2 0 8 6} \mathbf{~ c u}$ cms respectively.

Studies of lateral asymmetry of the legs have confirmed that the left leg is heavier than the right but the results of differences in length are not uniform (Latimer \& Lowrance 1965; Singh 1970). The dominance of the lower extremity is less marked than that of the upper. The dominant leg is expressed by the stronger tibia usually on the opposite side of the dominant $\mathrm{arm}^{11}$. In the present study right tali exhibited greater dimensions than left except breadth of talus. In a study by Harris SM, Case DT ${ }^{10}$ they observed that talus on the right side was generally exhibited greater dimensions than those from the left side. The asymmetry was less pronounced in case of tarsals and metatarsals. Structural asymmetry in fetal metatarsal bones on the right and left side was reported by Gawlikowska et al. (2007). In the younger group, the character of the asymmetry fluctuated. A clear one-sided domination in all metatarsal bones appeared only in the oldest group (over 26 gestational weeks). These results demonstrate that such asymmetry forms and matures during ontogenesis, similarly to other systems and organs (Gawlikowska etal. 2007). Asymmetry can be of genetic or nongenetic origin. Among the former type are the fundamental forms for which the signals present in the inherited genetic constitution produce definite right-left differences. The nongenetic forms of asymmetry can be determined by the influence exerted by the external environment, or they may be due to random developmental differences in the internal environment of the two halves of the body. The origin of lateralization involves both ontogenetic and phylogenetic factors (Babcock, 1993). The following factors are considered to be responsible for presence or absence of asymmetries in different structures of the body.

\section{Genes:}

A variety of genetic studies, including twin, family, adoption, and cross-fostering studies, demonstrated that handedness in humans has a significant genetic component (Arnold, 1844; Gennadis, 1858; Schultz, 1926,1937; Chamberlain, 1928; Pande \& Singh 1971; Singh 1971; Annett, 1974; Carter et al., , 1976; Hicks \& Hinsborne 1976; Longstreth,1980; McManus, 1991; Vettivel et al., 1995; Scheuer \& Black, 2007).

\section{Hormones:}

Majority of studies suggest that gonadal steroid hormones affect functional development during early ontogenesis (Murphy et al., 1994) Hormones appear to play a large role in mediating bodily asymmetry and phenotypic sex is related in systematic ways to cerebral asymmetry (de Courten-Myers, 1999; Frederikse et al., 1999; Kulynych et al., 1994; Moffat et al., 1998).

\section{Environmental Factors:}

Ability to change is one of the important propertiy of a living organism which is well visible in the course of individual life. Degree of asymmetry is a constant property in the process of ontogenesis where change in functional, dynamic and morphological asymmetry appearing with age (Wolanski, 1972). During childhood, the shape of the body continuously changes because of the different rates of growth of different parts. The gross changes in bodily proportions during growth in the human have been analyzed (Medawar, 1944). The degree of asymmetry reflects the degree of force exerted onto the right or left limb whereas the particular bone site showing the asymmetry indicates the kind of force exerted (Cuk et al., 2001). Vigorous training might change bone dimensions if this was undertaken before closure of the epiphyses has been completed (Tittle \& Wutscherk, 1992; Haapasalo et al., 2000). Asymmetry is also influenced by mechanical loading or disuse of a limb (Singh, 1971; Steele and Mays,1995).Use of dominant limb in athlete's results in greater asymmetry (Ruff \& Jones, 1981), while the activities involving non-dominant limb may change the asymmetry (Stirland, 1993). Activities involving both limbs equally may result in lack of asymmetry (Rule, 1982; Stirland, 1993). Protein deficiency diminishes the asymmetry as reported by Steyn \& Iscan (1999) and Mall et al. (2001). Though, right dominance is considered as congenital phenomenon (Pande \& Singh, 1971; Taylor JR \& Halliday, 1977), the dominance pattern could also be influenced by postnatal adaptation and physical work (Prives, 1960; Krishan, 2008) and can be enhanced or reduced according to individual's habits and activities, age, nutrition, overuse or disuse of the limb (Papaloucas et al. 2008). Industrial groups exhibit relatively less asymmetry than pre-industrial humans and less dimorphism in asymmetry. A mixture of influences from both 
genetic and behavioral factors is implicated as the source of these patterns (Auerbach \& Ruff, 2006). Adaptive development requires the organism to resist genetic and environmental stresses that disrupt the genetic plan for growth, a buffering capacity termed developmental stability (Thoma et al., 2002). One way to carefully assess how various stressors affect morphology is to investigate concomitant physiological changes in the body. For example, human experience elevated steroid hormone concentrations, namely glucocorticoids in response to stress. Glucocorticoids in turn determine how the body effectively copes with the stressors. These influences may not only affect immediate survival, but future growth, health, and reproduction (Handa et al., 1994; Ferin, 1999; Viau, 2002; Viau et al., 2005; Kudielka \& Kirschbaum,. 2005), all implicated as correlates of FA (Knierim et al., 2007). In addition, sex steroid hormones, namely androgens and estrogens are in part responsible for growth and reproduction (Kudielka \& Kirschbaum, 2005), and for the observed sex differences in response to stress (Kudielka \& Kirschbaum, 2005; Maclusky \& Naftolin 1981;Mc Ewen, 1981; Migeon \& Wisniewski, 1998). Several studies also linked androgens and estrogens to fluctuating asymmetry (Cunningham, 2000; Manning et al., 1997) ${ }^{11}$.

\section{Clinical Implications:}

The examination of the upper and lower limb asymmetries can be useful to medical scientists, archeologists, and anthropologists (Iscan \& Shihai 1995; King et al., 1998), to the policeand forensic experts and for medicolegal studies (Steyn \& Iscan 1999; Mall et al. 2001).

\section{Conclusion:-}

Body asymmetries indicate the developmental instability of an individual (Watson \& Thornhill 1994) and may indicate association with various disorders in the body. Knowledge and understanding of these asymmetries is important to achieve a good diagnosis and focus on an appropriate treatment and management plan. Evidence from behavioural, electrophysiological, and functional neuroimaging studies should be used to understand how these developmental disorders differ so that accurate differential diagnoses and appropriate targeted techniques can be effectively applied for remediation. Likewise, there are various etiological factors: genetic, environmental and functional to be well understood for a proper diagnosis and thus appropriate to focus on a plan treatment. Morphological parameters were different for right and left tali, where right tali exhibited greater dimensions compared to the left indicating the right tali is under more stress compared to left. Although this study shows the different in measurements for right and left tali but it is statistically insignificant.

\section{References:-}

1. Bilodi AK. Study of Calcaneal articular facets in human tali. Kathmandu Univ Med J 2006; 4:75-7.

2. Standring S. Gray's anatomy- The anatomical basis of clinical practice, $40^{\text {th }}$ edn., Spain: Elsevier Limited 2008:1429-62.

3. McPoil TG, Knecht HG. Biomechanics of the Foot in Walking: A Function Approach. J Orthop Sports Phys Ther 1985; 7:69-72

4. Sangeorzan BJ, Hansen ST Jr. Early and late post traumatic foot reconstruction. Clin Orthop Relat Res 1989; 243:86-91.

5. Ari I, Kafa IM. Bone length estimation and population specific features of calcaneus and talus bones of the late Byzantine Era. Coll Antropol 2009; 33(2): 613-8.

6. Mahato NK, Murthy SN. Articular and angular dimensions of the talus: interrelation and biomechanical significance. Foot 2012; 22(2):85-9.

7. Otag I, Cimen M. Morphometric measures of Talus bone in skeleton remains belonging to Anatolian Geography. Indian journal of applied research 2013; 3(8):530-31.

8. Gautham K, Clarista MQ, Sheela N and Vidyashambhava P. Morphometric analysis of the human tali. CIBTech Journal of Surgery 2013; 2(2):64-8.

9. Kuo CC, Lee GY, Chang CM, Hsu HC, Leardini A, Wu Lu T. Ankle morphometry in the Chinese population. Journal of Foot and Ankle Research: 2008; 1:011.

10. Harris SM, Case DT. Sexual Dimorphism in the tarsal bones: Implications for Sex Determination. J Forensic Sci 2012; 57(2): 295-305.

11. Zeenat FZ. Body asymmetries: incidence. Etiology and clinical implications. Australian Journal of Basic and Applied Science, 2011; 5(9): 2157-91. 